-Research Note-

\title{
Histological and Absorptional Changes of Skin with Development of Rat Fetuses
}

\author{
Akira NARITA, Masahiko KUMAKURA ${ }^{1)}$, Sadami SHIBABE ${ }^{2)}$ \\ Masayuki ITO2), Akiyo SHIGEMATSU and Toshio NANBO ${ }^{3)}$
}

\author{
Institute of Whole Body Metabolism, Chiba 270-1407, \\ ${ }^{1)}$ Department of Anatomy, School of Dentistry at Niigata, The Nippon Dental \\ University, Niigata 951-8580, \\ 2)Japan Atomic Energy Research Institute, Tokyo 113-0021, and \\ ${ }^{3)}$ Institute of Xenobiotic analysis, Chiba 270-1402, Japan
}

\begin{abstract}
Immature epidermis, dermis and subcutaneous tissues were histologically observed in the fetal skin of rats on day 17 of pregnancy. The tissues had become thicker in the fetal skin by day 19, and formed the basement membrane. The strata corneum and granulosum were found in the fetal skin on day 21. The distance between the cutaneous surface and the vascular system increased with fetal development. None of the fetal skin contained any subcutaneous adipose tissue or sebaceous glands as in the maternal ones. Absorption ratios of radioactivities in the rat fetuses were assayed at $20 \mathrm{~min}$ after injecting ${ }^{14} \mathrm{C}$-phenol and ${ }^{14} \mathrm{C}$-4-tert-butylphenol into the amniotic fluid. The absorption of both compounds decreased with fetal development. The absorption of ${ }^{14} \mathrm{C}$-phenol was greater than that of ${ }^{14} \mathrm{C}$-4-tert-butylphenol in the rat fetuses in three stages. These results suggest that the decrease in the absorption with fetal development depends on the increase in the distance between the cutaneous surface and the vascular system, and on the formation of the stratum corneum. The lower absorption of the ${ }^{14} \mathrm{C}-4$-tert-butylphenol having high hydrophobicity could be because the fetal skin was without subcutaneous adipose tissue and sebaceous glands.
\end{abstract}

Key words: Histology, Absorption, Fetal Rat, Skin.

(J. Reprod. Dev. 44: 205-208, 1998)

E xposure of the fetus to chemicals is influenced by various steps: placental transfer, distribution in the fetal tissue, metabolism and transfer between the fetal body and amniotic fluid [1,2]. A previous report [2] showed that the transfer of chemicals between the fetal body and amniotic fluid is mainly via the fetal skin, and changes with fetal development, but apparently no histological report on the fetal skin in rats has been published to date. The present study attempted to relate the transfer of phenolic compounds to the structure of

Accepted for publication: February 27, 1998

Correspondence: A. Narita, Biomedical Research Institute, Chiba 270-1402, Japan the fetal skin, by using rats on days 17, 19 and 21 of pregnancy.

\section{Materials and Methods}

Thirty female rats of the Wistar strain were used on days 17, 19 and 21 of pregnancy. They were kept at $24 \mathrm{C}$ under a $14 \mathrm{~h}$ lighting condition $(0400$ $\mathrm{h}-1800 \mathrm{~h}$ ), and were caged with males for mating at proestrus at 10-12 weeks of age. The morning when spermatozoa were detected in the vaginal smear was defined as day one of pregnancy.

The fetal and maternal rump skins were then 
immediately removed under anesthesia, fixed in Bouin solution, dehydrated with ethyl alcohol, embedded in paraffin, sectioned at a thickness of 3 $\mu \mathrm{m}$, and stained with hematoxylin and eosin.

A small incision was made in the abdomen, and ${ }^{14} \mathrm{C}$-phenol and ${ }^{14} \mathrm{C}$-4-tert-butylphenol were injected into the amniotic fluid through the uterine wall as described in a previous report [3]. At $20 \mathrm{~min}$ after the injection, the amniotic fluid was extracted, and the fetus was immediately washed in saline solution at $37 \mathrm{C}$. The amniotic fluid and saline solution were combined, followed by the measurement of the radioactivity. It was preliminarily

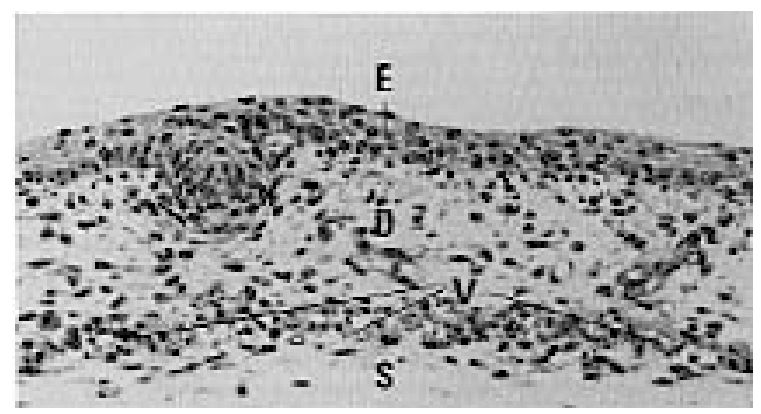

Fig. 1. Fetal rat skin containing epidermis (E), dermis (D), subcutaneous tissue (S) and vascular system (V) on day 17 of pregnancy. H-E staining. $\times 200$.

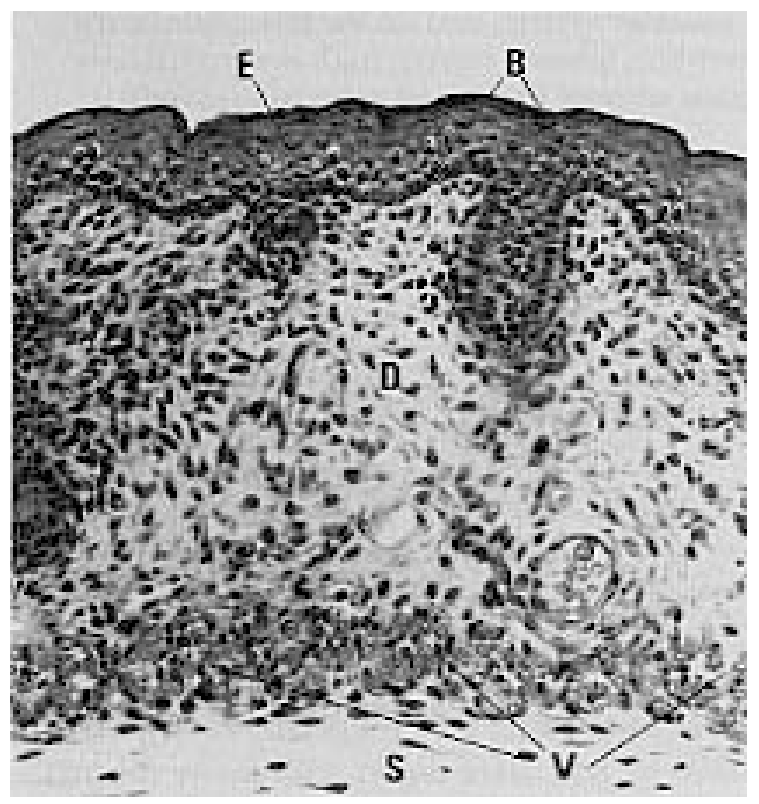

Fig. 2. Fetal rat skin containing epidermis (E), dermis (D), subcutaneous tissue (S), basement membrane (B) and vascular system (V) on day 19 of pregnancy. H-E staining. $\times 200$. confirmed that both labelled compounds in the amniotic fluid decreased linearly until $30 \mathrm{~min}$ after the injection; thus we assayed the absorption ratio at $20 \mathrm{~min}$. The partition coefficient $(\log \mathrm{P})$ was determined by the method described in a previous report [4].

\section{Results}

The fetal and maternal skins were histologically observed in rats on days 17, 19 and 21 of pregnancy. The immature epidermis, dermis and subcutaneous tissue were observed in the fetal skin on day 17 (Fig. 1). The tissues became thicker in the fetal skin on day 19, and formed the basement membrane (Fig. 2). The strata corneum and granulosum containing keratohyalin granules were found in the fetal skin on day 21 (Fig. 3). The

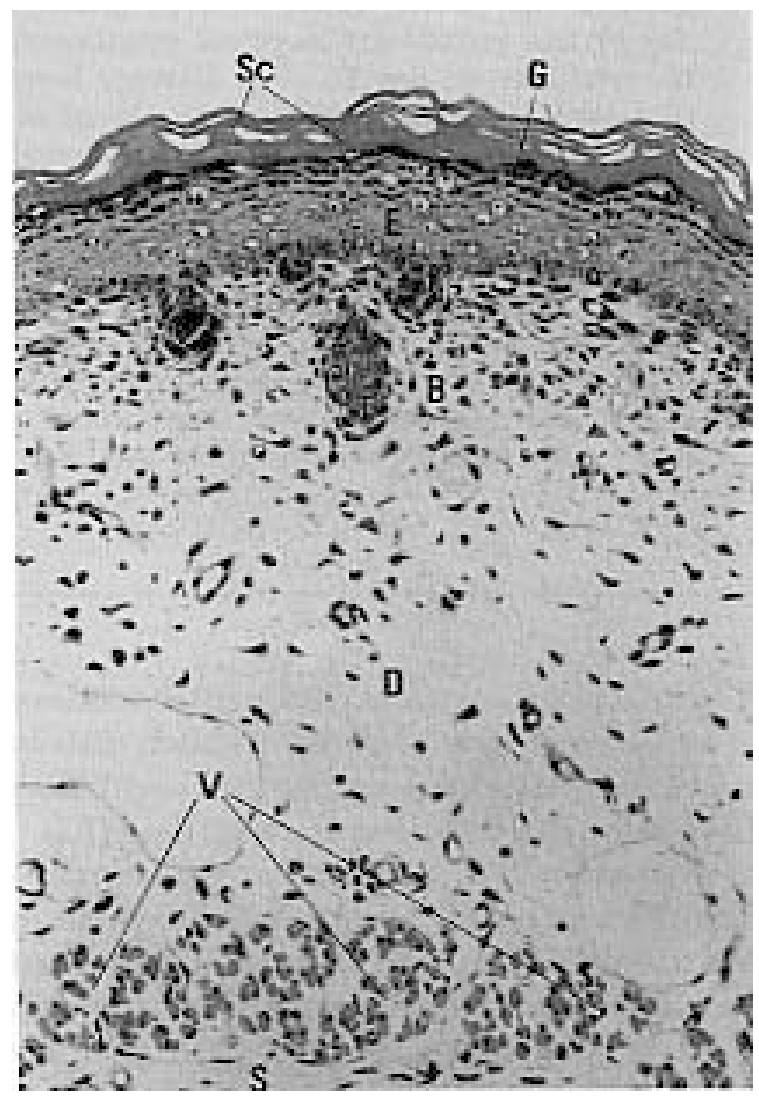

Fig. 3. Fetal rat skin containing epidermis (E), dermis (D), subcutaneous tissue (S), basement membrane (B), strata corneum $(\mathrm{Sc})$, granulosum $(\mathrm{G})$ and vascular system $(\mathrm{V})$ on day 21 of pregnancy. H-E staining. $\times 200$. 
distance between the cutaneous surface and the vascular system increased with fetal development. Each fetal skin contained no subcutaneous adipose tissue or sebaceous glands as in the maternal ones (Fig. 4).

Absorption ratios of ${ }^{14} \mathrm{C}$-phenol and ${ }^{14} \mathrm{C}$-4-tertbutylphenol in the rat fetuses on days 17, 19 and 21 of pregnancy are shown in Table 1 . The absorption of both compounds decreased with fetal development. The absorption of ${ }^{14} \mathrm{C}$-phenol was greater than that of ${ }^{14} \mathrm{C}$-4-tert-butylphenol in the rat fetuses in the three stages. The values for $\log \mathrm{P}$ of both phenol and 4-tert-butylphenol were 1.53 and 2.92 , respectively.

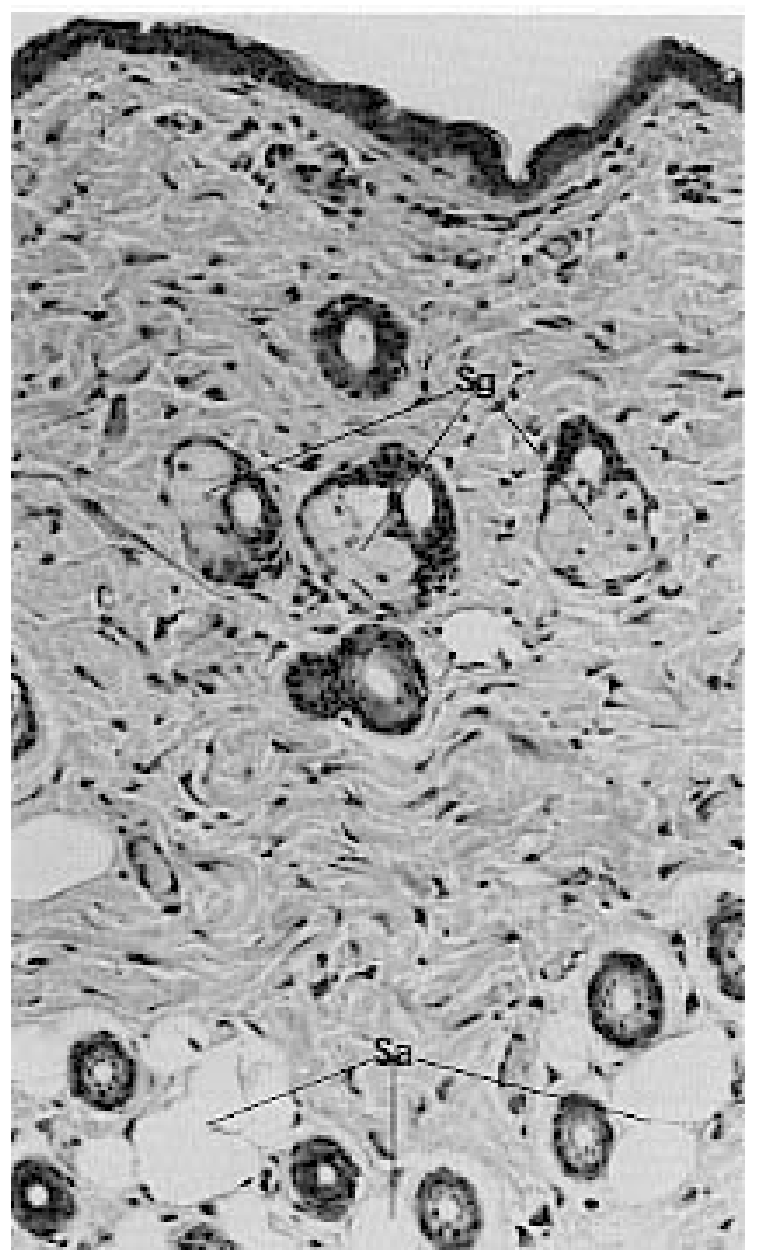

Fig. 4. Maternal rat skin containing subcutaneous adipose tissues (Sa) and sebaceous glands (Sg). H-E staining. $\times 200$.
Table 1. Ratios of absorption of ${ }^{14} \mathrm{C}$-phenol and ${ }^{14} \mathrm{C}$-4-tertbutylphenol from the amniotic fluid into the fetal body

\begin{tabular}{|c|c|c|}
\hline \multirow[b]{2}{*}{ Gestation day } & \multicolumn{2}{|c|}{ Absorption ratios ( $\%$ of amount injected) } \\
\hline & Phenol & 4-tert-butylphenol \\
\hline 17 & $51.2 \pm 5.8$ & $35.3 \pm 4.6$ \\
\hline 19 & $45.6 \pm 4.9$ & $28.5 \pm 3.3$ \\
\hline 21 & $22.7 \pm 2.9$ & $5.8 \pm 0.8$ \\
\hline
\end{tabular}

Deta are the mean \pm S.E. for four or five animals.

\section{Discussion}

It was thought that substances in the amniotic fluid were absorbed into fetal body through the digestive tract and lung as well as the skin. Nanbo [2], using whole body autoradiography, showed that a labeled compound injected into the amniotic fluid of the rat on day 21 of pregnancy was not observed in the esophagus and the trachea, but was distributed in the tissues within $60 \mathrm{~min}$. From these results, Nanbo [2] concluded that substances in the amniotic fluid were absorbed from the skin within $60 \mathrm{~min}$. Thus it is assumed that the present data reflect the absorption from the fetal skin.

In our experiments, the decrease of absorption ratios of phenol and 4-tert-butylphenol with fetal development could possibly be related to the distance between the cutaneous surface and the vascular system, because development of the epidermis without the vascular system must inhibit the absorption. A rapid decrease in the absorption ratios for the two compounds in the fetal skin of rats on Day 21 of pregnancy may be attributed to the formation of the stratum corneum as a barrier.

The absorption ratios of hydrophobic compounds are generally higher than those of hydrophilic compounds in the skin $[5,6]$ because biological membranes consist of hydrophobic phospholipids and proteins [7]. However, our experiments showed an opposite result: 4-tert-butylphenol with hydrophobic properties (log P: 2.92) was absorbed at a lower rate than phenol with hydrophilic properties ( $\log$ P: 1.53). This discrepancy may be because the fetal skin contains no subcutaneous adipose tissue or sebaceous glands, making absorption of hydrophobic compounds in the fetal skin difficult, or alternatively, hydrophilic compounds may easily pass through the cutaneous tissues of rat fetuses. 


\section{References}

1. Mirkin BL, Singh S. Placental transfer of pharmacologically active molecules. In: Mirkin BL (ed.), Perinatal Pharmacology and Therapeutics. New York: Academic Press; 1976: 1-69.

2. Nanbo T. Studies on the absorption mechanism for the glucuronide of p-phenylbenzoic acid in fetal compartment. J Pharm Dyn 1982; 5: 988-993.

3. Nanbo T. Pharmacokinetics in maternal-fetal unit after intravenous administration of $p$ phenylbenzoic acid to rat. J Pharm Dyn 1982; 5: 213-221.

4. Nanbo T. The effect of 4-substituent of benzoic acid on glycine conjugation. Chem Pharm Bull 1990; 38: 1753-1754.
5. Austel V, Kutter E. Absorption, distribution, and metabolism of drugs. In: Topliss JG (ed.), Quantitative Structure-Activity Relationships of Drugs. New York: Academic Press; 1983: 437-496.

6. Stinchcomb AL, Paliwal A, Dua R, Imoto $\mathbf{H}$, Woodard RW, Flynn GL. Permeation of buprenorphine and its 3-alkylester prodrugs through human skin. Pharmaceutical Research 1996; 13: 1519-1523.

7. Blankley CJ. Introduction: a review of QSAR methodology. In: Topliss JG (ed.), Quantitative Structure-Activity Relationships of Drugs. New York: Academic Press; 1983: 1-21. 